\title{
Systemic Therapy for Combined Hepatocellular-Cholangiocarcinoma: A Single-Institution Experience
}

\author{
Nikolaos A. Trikalinos, MDa, ; Amy Zhou, MD ${ }^{a,} ;$; Maria B. Majella Doyle, MD, MBA, FRCS ${ }^{b}$; \\ Kathryn J. Fowler, MD; Ashley Morton, NPa; Neeta Vachharajani, CRC ${ }^{\mathrm{b}}$; Manik Amin, $\mathrm{MD}^{\mathrm{a}}$; \\ Jesse W. Keller, MD; ; William C. Chapman, MD ; Elizabeth M. Brunt, MD; and Benjamin R. Tan, MDa
}

\begin{abstract}
Background: Combined hepatocellular-cholangiocarcinoma tumors (CHCC-CCA) are a heterogeneous group of rare malignancies that have no established optimal treatment. Patients and Methods: We identified patients with cHCC-CCA treated at a tertiary center and retrospectively examined their histology, interventions, and outcomes. We calculated disease control rate (DCR), disease progression, overall survival, and progression-free survival (PFS) between treatment subgroups. Results: A total of 123 patients were evaluable. Interventions included resection, locoregional therapy, transplant, chemotherapy, and targeted agents. Ultimately, 68 patients received systemic treatment-57 with gemcitabine plus either 5-fluoropyrimidine (5-FU) or a platinum combination. Disease progression was more common in the gemcitabine/5-FU group versus gemcitabine/platinum ( $P=.028)$, whereas DCR favored gemcitabine/platinum (78.4\% vs $38.5 \%$; $P=.0143$ ). Median PFS from time of initial diagnosis favored the gemcitabine/platinum group, but the difference did not reach statistical significance. Targeted agents had minimal to no effect on survival metrics. Conclusions: Gemcitabine/platinum seems to be a superior regimen for patients with CHCC-CCA who require systemic treatment. Further studies are needed to clarify the regimen's efficacy and applicability in patient subgroups.
\end{abstract}

Combined hepatocellular-cholangiocarcinomas (cHCCCCAs) represents a heterogeneous group of primary liver carcinomas with both biliary and hepatocellular differentiation. ${ }^{1,2}$ These tumors are rare, accounting for approximately $1 \%$ to $14 \%$ of all primary liver cancers. ${ }^{2}$ cHCCCCAs were first reported in 1903 by Wells ${ }^{3}$ and further characterized by Allen and Lisa in 1949. ${ }^{4}$

Currently, there is no consensus terminology for these malignancies; they have been referred to by several names, including hepatocholangiocellular carcinoma, mixed HCC-CCA, and biphenotypic (hepatobiliary) primary liver carcinomas. cHCC-CCAs are

\footnotetext{
Departments of a Medicine, bSurgery, 'Radiology, and dPathology and Immunology, Washington University in St. Louis, St. Louis, Missouri.

*These authors contributed equally.

Submitted January 25, 2018; accepted for publication June 11, 2018.

The authors have disclosed that they have no financial interests,

arrangements, affiliations, or commercial interests with the manufacturers

of any products discussed in this article or their competitors.
}

increasingly recognized as distinct from hepatocellular carcinomas (HCCs) and intrahepatic cholangiocarcinomas (ICCs). In 2010, WHO classified them into 2 categories: (1) the more commonly encountered classical cHCC-CCA with coexistent HCC and cholangiocarcinoma histology, and (2) 3 variants of cHCC-CCA with stem cell features. ${ }^{5,6}$ Advances in histopathologic characterization have revealed much greater complexity than initially described, and categorization of these tumors continues to evolve. ${ }^{1}$

Patients with cHCC-CCA have a worse prognosis than those with pure HCC or ICC. ${ }^{7-10}$ Surgical

Author contributions: Protocol concept: Zhou, Tan. Data extraction and editing: Zhou, Trikalinos, Tan, Vachharajani. Manuscript preparation, revision, and final approval: All authors.

Correspondence: Nikolaos A. Trikalinos, MD, Department of Medicine, Division of Oncology, Washington University in St. Louis, 660 South Euclid Avenue, Campus Box 8056-29, St. Louis, MO 63110

Email: ntrikalinos@wustl.edu 
Trikalinos et al

resection appears to offer the best chances for survival, but only a minority of patients are eligible. ${ }^{11}$ For unresectable lesions, locoregional therapy (LRT) or systemic therapy is considered as per experience with other malignancies. ${ }^{11}$ Unfortunately, given the rarity of cHCC-CCAs, there are no generally established guidelines or standard treatments. This is inherently problematic, because treatment of ICC and HCC ranges from oral targeted agents, such as sorafenib, ${ }^{12}$ to intravenous cytotoxic chemotherapy. ${ }^{13,14}$

This report details the characteristics and outcomes of 68 patients with unresectable or recurrent cHCC-CCA treated with cytotoxic chemotherapy or targeted agents.

\section{Patients and Methods}

\section{Patients}

We conducted a retrospective chart review under an Institutional Review Board-approved Washington University Liver Cancer Registry. The registry has a retrospective and prospective component and collects information on consenting patients with various liver pathologies. We identified 123 patients with histopathologically proven cHCC-CCA in the Department of Pathology database at Washington University treated between 1999 and 2016. One of the main objectives of the study was to identify patients with pathologically confirmed cHCC-CCA who received systemic therapy for unresectable disease or disease recurrence after surgery and/or liver-directed LRT. Clinical information was obtained from review of patient electronic charts.

\section{Outcomes}

Recorded outcomes were overall response rate, disease control rate (DCR), progression-free survival (PFS), and overall survival (OS). Response to therapy was based on radiographic data from CT or MRI as determined by RECIST criteria. ${ }^{15}$ DCR was defined as the percentage of patients who achieved complete response, partial response, or stable disease with systemic treatment. PFS was defined as the interval from first-line therapy to time of disease progression. OS was defined as the interval from time of pathologic diagnosis to the study end point (eg, death, if applicable, or last follow-up).

\section{Pathology and Immunohistochemistry}

Pathologic classification of cHCC-CCA was determined by light microscopy showing biphenotypic/ mixed hepatobiliary histologic features and immunohistochemistry demonstrating features of both biliary and hepatocellular differentiation. Biliary differentiation was suggested by staining with $\mathrm{K} 7$ and K19. Hepatocellular differentiation was based on polyclonal carcinoembryonic antigen and CD10 with canalicular staining and cytoplasmic hepatocyte paraffin 1 (HepPar1). All slides were evaluated in consensus with an experienced hepatopathologist (E.M.B.).

\section{Statistical Analysis}

Statistical analyses were performed using GraphPad Prism, version 5.0 (GraphPad Software, Inc.). Categorical variables were compared using Fisher exact test or chi-square test as applicable. Student $t$ test or Mann-Whitney U test was used, as applicable, to compare continuous variables. OS and PFS were plotted using Kaplan-Meier curves, and survival functions were compared using the log-rank test. A 2 -sided $P$ value of $<.05$ was considered statistically significant.

\section{Results}

\section{Patient Characteristics}

Baseline characteristics for all patients are summarized in Table 1. Diagnosis was made from liver biopsy in $60 \%$ of patients $(n=74)$, resection in $28 \%(n=34)$, and explant in $10 \%(n=13)$, and $2 \%$ of diagnoses $(n=3)$ were diagnosed based on lymph node/vertebral biopsies. The HCC/ICC ratio was not estimated on tumor samples. Our cohort included mainly Caucasians, with approximately 10\% African Americans and only approximately 3\% Asians. Median age at diagnosis was 62 years (range, 21-89 years), maleto-female ratio was $1.4: 1$, and no patients had previously received any systemic cytotoxic treatment; $39 \%$ of the patients were obese (body mass index [BMI], $>30 \mathrm{~kg} / \mathrm{m}^{2}$ ) and an additional $33.3 \%$ patients were overweight (BMI, 25-30 kg/m²). Less than 35\% had a diagnosis of cirrhosis based on pathology and imaging review. A total of 21 patients were hepatitis $\mathrm{C}$ virus ( $\mathrm{HCV}$ )-positive and 9 were hepatitis $\mathrm{B}$ virus (HBV)-positive; testing was not performed on 45 patients. There were no coexistent $\mathrm{HBV} / \mathrm{HCV}$ viral 
Systemic Therapy for Biphenotypic Cholangiocarcinoma

\begin{tabular}{|c|c|c|c|c|}
\hline & $\begin{array}{l}\text { All Patients } \\
(\mathrm{N}=123)\end{array}$ & $\begin{array}{l}\text { No Systemic Therapy } \\
\qquad(\mathrm{N}=55)\end{array}$ & $\begin{array}{l}\text { Systemic Therapy } \\
\qquad(\mathrm{N}=68)\end{array}$ & $P$ Value \\
\hline Median age at diagnosis (range), y & $62(21-89)$ & $61(26-89)$ & $60(21-82)$ & .7241 \\
\hline Male:Female & $71: 52$ & $38: 17$ & 33:35 & .0278 \\
\hline Ethnicity & & & & .0452 \\
\hline Caucasian & 109 & 45 & 64 & \\
\hline African American & 11 & 7 & 4 & \\
\hline Asian & 3 & 3 & - & \\
\hline $\mathrm{BMI}, \mathrm{kg} / \mathrm{m}^{2}$ & & & & .7203 \\
\hline$<25$ & $31(25.2 \%)$ & $10(18.2 \%)$ & $21(30.9 \%)$ & \\
\hline $25-30$ & $41(33.3 \%)$ & $20(36.4 \%)$ & $21(30.9 \%)$ & \\
\hline$>30$ & $48(39.0 \%)$ & $22(40.0 \%)$ & $26(38.2 \%)$ & \\
\hline No data & $3(2.4 \%)$ & $3(5.5 \%)$ & $0(0 \%)$ & \\
\hline Cirrhosis & & & & .0018 \\
\hline Yes & $40(32.5 \%)$ & $26(47.3 \%)$ & $14(20.6 \%)$ & \\
\hline No & $79(64.2 \%)$ & $27(49.1 \%)$ & $52(76.5 \%)$ & \\
\hline No data & $4(3.3 \%)$ & $2(3.6 \%)$ & $2(2.9 \%)$ & \\
\hline OLT & $13(10.6 \%)$ & $12(21.8 \%)$ & $1(1.5 \%)$ & .0005 \\
\hline Resection & $40(32.5 \%)$ & $23(41.8 \%)$ & $\begin{array}{c}18(26.5 \%) \\
\text { (11 before systemic } \\
\text { therapy; } 7 \text { after) }\end{array}$ & .0853 \\
\hline Median largest tumor diameter (range), cm & $6.6(1.0-19.0)$ & $4.9(1.0-16.0)$ & $7.7(1.0-19.0)$ & .0194 \\
\hline Positive lymph nodes & $69(56.1 \%)$ & $21(38.2 \%)$ & $48(70.6 \%)$ & .0003 \\
\hline Distant metastasis & $30(24.4 \%)$ & $7(12.7 \%)$ & $23(33.8 \%)$ & .0068 \\
\hline
\end{tabular}

Unless otherwise specified, results are reported as medians with range and $\mathrm{N}(\%)$.

Abbreviations: BMI, body mass index; OLT, orthotopic liver transplant.

infections in the patient pool. A total of 8 patients who were $\mathrm{HCV}$-positive and 4 who were HBV-positive received systemic therapy.

\section{Treatments}

Of the 123 patients, $55(44.7 \%)$ did not receive any systemic therapy. A total of 23 (19\%) underwent resection and $12(10 \%)$ underwent orthotopic liver transplantation (OLT) with curative intent; of the remaining patients who did not undergo systemic therapy $(n=20)$, most were placed in hospice or died within 6 months.

Of the 68 patients who received systemic therapy, 11 also received LRT with transarterial chemoembolization (TACE), yttrium-90 (Y90), hepatic artery infusion (HAI) pump, or a combination before starting chemotherapy. Choice of treatment, including LRT or chemotherapy, was based on physician preference. A total of 11 patients had undergone resection prior to chemotherapy (1 combined with
TACE), but all experienced recurrence at an average interval of $9.6( \pm 8.7)$ months.

Most patients who received chemotherapy were ultimately treated with a gemcitabine-based regimen (57/68); 16 (23.5\%) received gemcitabine alone or in combination with a fluoropyrimidine (5-FU) based on patient and physician discretion and 41 $(60.3 \%)$ received gemcitabine in combination with a platinum drug (cisplatin or oxaliplatin). A total of 7 patients were treated with sorafenib, 3 were treated with 5 -FU alone, and 1 was treated with erlotinib plus bevacizumab.

\section{Response to Gemcitabine-Based Treatments}

A total of $88 \%$ of patients (50/57) who received a gemcitabine-based regimen were evaluable for response (Table 2), including 37 of 41 patients who received gemcitabine/platinum combination and 13 of 16 who received gemcitabine \pm 5 -FU. None of the patients achieved a complete remission. Patient responses were categorized as partial response, stable 
Trikalinos et al

Table 2. Treatment Outcomes

\begin{tabular}{|c|c|c|c|c|}
\hline \multirow[b]{2}{*}{ Response } & \multicolumn{3}{|c|}{$\begin{array}{c}\text { Gemcitabine-Based } \\
(\mathrm{N}=57)\end{array}$} & \multirow[b]{2}{*}{$\begin{array}{c}\text { Sorafenib } \\
(\mathrm{N}=7)\end{array}$} \\
\hline & $\begin{array}{l} \pm \mathrm{FU} \\
(\mathrm{n}=16)\end{array}$ & $\begin{array}{c}+ \text { Platinum } \\
(n=41)\end{array}$ & $P$ Value & \\
\hline Evaluable for response & 13 & 37 & & 5 \\
\hline Partial response & $2(15.4 \%)$ & $9(24.3 \%)$ & \multirow{3}{*}{.0281} & 0 \\
\hline Stable disease & $3(23.1 \%)$ & $20(54.1 \%)$ & & $1(20 \%)$ \\
\hline Disease progression & $8(61.5 \%)$ & $8(21.6 \%)$ & & $4(80 \%)$ \\
\hline DCR & $38.5 \%$ & $78.4 \%$ & .0080 & $20 \%$ \\
\hline Median PFS, mo & 6.6 & 8.0 & .3280 & 4.8 \\
\hline Median OS, mo & 11.7 & 11.5 & .9686 & 9.6 \\
\hline
\end{tabular}

Unless otherwise specified, results are reported as medians with range and $\mathrm{N}(\%)$.

Abbreviations: DCR, disease control rate; OS, overall survival; PFS, progression-free survival.

disease, and disease progression. The 2 subgroups were significantly different $(P=.028)$, with disease progression more common in patients receiving gemcitabine \pm 5 -FU $(61.5 \%)$ than those receiving gemcitabine/platinum (21.6\%).

DCR was markedly different between patients who received a gemcitabine/platinum combination (29/37) and those receiving gemcitabine \pm 5-FU (5/13), favoring the former $(78.4 \%$ vs $38.5 \%$; $P=.008)$ with a relative risk of 1.71 (95\% CI, 1.032.84). A multivariate analysis using age ( $>60$ years), BMI $\left(>30 \mathrm{~kg} / \mathrm{m}^{2}\right)$, prior LRT, largest tumor diameter $(>5 \mathrm{~cm})$, and type of systemic therapy as input variables showed that type of therapy was the only variable significantly affecting DCR $(P=.013)$. Patients receiving gemcitabine/platinum were more likely to experience disease control (hazard ratio, 7.14; 95\% CI, 1.54-33.3).

Time to progression was comparable in the 2 gemcitabine-based groups $(6.1 \pm 4.2$ months for gemcitabine/platinum vs $5.4 \pm 6.6$ months for gemcitabine $\pm 5-\mathrm{FU} ; \mathrm{P}=.98)($ Figure 1$)$.

\section{Response to Other Agents}

Among the 7 patients treated with sorafenib, 5 were evaluable for treatment response (Table 2); 4 patients had disease progression, and only 1 had stable disease. Among the 3 patients who received 5-FU-based therapy, 1 normal-weight patient with minimally metastatic disease was treated with capecitabine, experienced clinical progression, and died 1.5 months after diagnosis. A 65-year-old overweight male patient with no underlying liver disease and with a $10.1-\mathrm{cm}$ tumor and 1 satellite lesion that was initially unresectable received floxuridine via
HAI. He experienced a partial response and later underwent resection of his tumor, surviving a total of 21.9 months. A 71-year-old obese female patient with a single $1-\mathrm{cm}$ lesion was treated with $5-\mathrm{FU}$ in conjunction with radiation. She declined further therapy and did not experience progression for $>50$ months until the end of the study period. A 77-yearold normal-weight female patient with a $3.4-\mathrm{cm}$ solitary tumor and no underlying liver disease was treated with erlotinib and bevacizumab, showed a complete radiographic response, and has not experienced progression $>4$ years after diagnosis. ${ }^{16}$

\section{Overall Survival (Median)}

Median OS for all 123 patients was 15.4 months; for the 68 patients ultimately receiving systemic therapy it was 12.1 months. For the 41 patients receiving gemcitabine/platinum therapy, OS was 11.5 months versus 11.7 months for the 16 patients

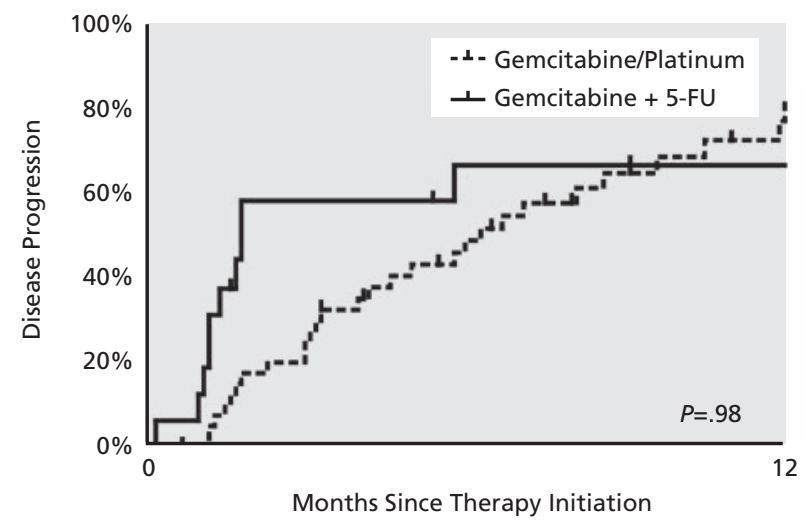

Figure 1. Time to progression from the initiation of therapy in patients receiving gemcitabine-based therapy $(P=.98)$.

Abbreviation: 5-FU, 5-fluoropyrimidine. 
receiving gemcitabine \pm 5 -FU $(P=.9686)$. A total of 47 patients received systemic therapy without any prior LRT or resection, and had a median survival of 9.1 months. Patients who received no surgery or systemic therapy only survived 5.1 months.

We sought to examine the effect of prior LRT (TACE, Y90, or HAI) or resection in the 68 patients who ultimately received systemic therapy; respective median OS was 16.3 and 17.9 months, respectively, which was nonsignificant $(P=.0678)$.

We compared patients who received previous resection followed by chemotherapy with those who only received systemic chemotherapy, and found their OS was significantly different (17.9 vs 9.1 months, respectively; $P=.0389$ ). This effect was lost, however, when OS was measured from the time of recurrence for the resection group (9.7 vs 9.1 months, respectively; nonsignificant).

A total of 41 patients ultimately received gemcitabine/platinum-based therapy. Of these, $5 \mathrm{had}$ undergone prior LRT, 5 had received resection, and 31 were treated with chemotherapy only. OS and median survival in the 3 groups were statistically comparable.

A total of 47 patients did not receive any pretreatment in the form of LRT or resection and were considered treatment-naïve. Of these, 31 received gemcitabine/cisplatin therapy, whereas 16 received other systemic chemotherapy; median OS was 9.7 versus 7.2 months, favoring gemcitabine/cisplatin, but was not statistically significant $(P=.4026)$.

\section{Progression-Free Survival}

Median PFS from initial diagnosis favored gemcitabine/platinum over gemcitabine/5-FU (8.0 vs 6.6 months) but was not statistically significant (Figure 2; $P=.33$ ). For sorafenib monotherapy, PFS was only 4.8 months.

\section{Discussion}

Mixed histology HCCs are rare, comprising a small percentage of primary liver tumors. Our institutional experience with $>120$ cases, $64 \%$ of them noncirrhotic, sheds some light on their prevalence and treatment challenges.

The epidemiology of patients with cHCCCCA may differ by geographic region. A retrospective cohort study reported on the characteristics of

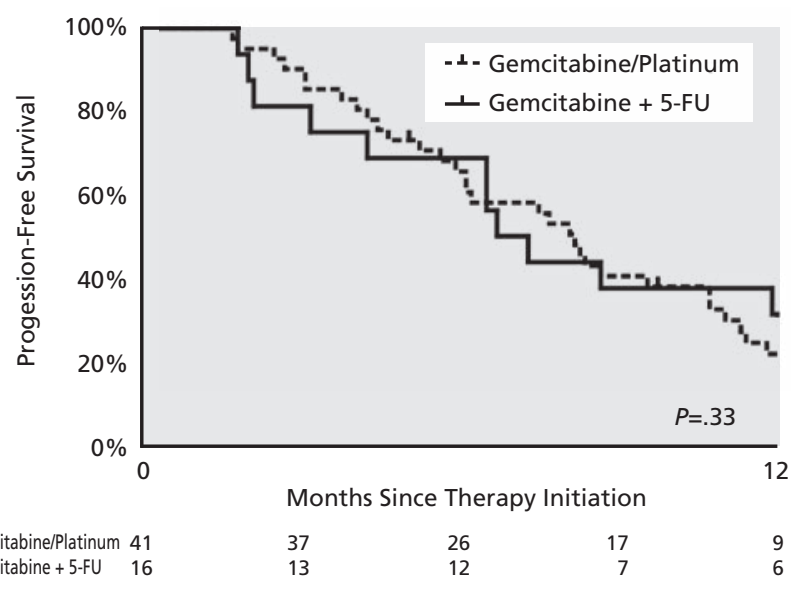

Figure 2. Progression-free survival in patients with combined hepatocellular-cholangiocarcinoma who received gemcitabine-based therapy $(P=.33)$.

Abbreviation: 5-FU, 5-fluoropyrimidine.

45 Chinese patients with cHCC-CCA ${ }^{17}$ and found that these tumors were more frequently observed in middle-aged male patients with chronic hepatitis or cirrhosis. Chronic HBV and HCV were also found to be major causes of cHCC-CCA in another retrospective cohort study of 65 patients from Taiwan. ${ }^{18}$ In contrast, our study demonstrated a balanced male-to-female ratio, and only a minority of patients (33\%) had cirrhosis, which is similar to previously published results from the United States. ${ }^{2}$ This could indicate a regional (United States vs Asia) variation. We only had hepatitis serologies on 33 of 68 treated patients, thus no comparisons between Asian and US patients were made. We observed that more than two-thirds of the patients in our cohort were overweight or obese, suggesting that this may be a potential risk factor for $\mathrm{cHCC}-\mathrm{CCA}$; nonalcoholic steatohepatitis was present in only 1 patient. This is in accordance with prior studies that have confirmed an association between obesity and an increased risk of CCA and HCC. 19,20 $^{2}$

There is no consensus on treatments for $\mathrm{cHCC}$ CCA given their rarity, and current approaches are extrapolated from what has shown efficacy in HCC or ICC. Hepatic resection, when possible, remains the preferred treatment and may offer patients the longest OS. ${ }^{11}$ However, the risk of recurrence is high even after resection with clear margins, with a median time to recurrence of 6 to 9 months, ${ }^{10}$ and patients tend to do poorly with salvage treatments.

At our institution, biopsy is performed in patients with radiologic characteristics not totally 
consistent with HCC (LI-RADS 5) and who have elevated CA 19-9/ $\alpha$-fetoprotein levels. If the biopsy specimen is confirmed by pathology to be a biphenotypic tumor, the patient is considered for resection/liver transplant after discussion in a multidisciplinary tumor board. LRT may be offered for patients with liver-only disease. For patients with metastatic or unresectable tumors, we offer systemic treatment with platinum and gemcitabine, and send the tumor samples for genomic analysis in an attempt to identify actionable mutations, including mismatch repair (MMR) protein status, IDH1, and others. Immunotherapy can be used for microsatellite instability-high (MSI-H) patients. With the approval of second-line nivolumab in HCC, use of checkpoint inhibitors for this rare biphenotypic tumor needs to be further studied.

In our cohort of 123 patients, we were able to confirm the poor prognosis of cHCC-CCA and showed a median OS of $<1$ year, including subgroups with previous surgical resection and/or systemic therapy. For patients with advanced disease requiring systemic chemotherapy, gemcitabine-based regimens were used in most, as per standard first-line treatment for patients with advanced ICC. ${ }^{14,21}$ In published studies, the combination of gemcitabine and cisplatin has produced a median OS of 11.7 months and a DCR of $81.4 \% .{ }^{14}$ Our observations were consistent with those in the current literature, with a median OS and response rate of 10.9 months and $78.4 \%$, respectively. Gemcitabine alone or in combination with 5-FU demonstrated lower overall response rates and DCRs compared with combination gemcitabine/platinum, which is also consistent with what has been reported for biliary tract cancers. ${ }^{14}$ Gemcitabine/platinum combination seemed superior to other chemotherapies in treatment-naïve patients, but our sample size did not allow for demonstration of statistical significance. Moreover, patients often received second-line treatments, sometimes with local oncologists, diluting the effect on survival metrics. Therefore, no definite conclusions can be drawn.

Our population cohort was heterogeneous, which affected OS and PFS metrics. Of the 55 patients who did not receive chemotherapy, 35 had undergone hepatic resection $(n=23)$ or OLT $(n=12)$ with curative intent. Thus, their prognosis is expected to be better than those with unresectable disease, affecting the median OS of the group. Indeed, of the 19 evaluable patients at 36 months, 12 had not received any treatment. Patients in the sorafenib group demonstrated a lower PFS, but later lines of treatment probably affected OS as well. The DCR and PFS favored a gemcitabine/platinum regimen, and subgroup analysis shed more light into the differences between treatment approaches. However, despite the significant effort to put together this database of rare tumors, we still had limited numbers of subjects who underwent multiple treatment sequences, which did not allow for meaningful comparisons or any form of matching. Our data also likely model the variable natural history of cHCC-CCA and the significant heterogeneity between these tumors, which can affect response to treatments.

HCCs are generally not responsive to chemotherapy. Sorafenib, an oral multikinase inhibitor of vascular endothelial growth factor receptor, plateletderived growth factor receptor, and Raf, has demonstrated efficacy with improvement in median OS (10.7 months) compared with placebo (7.9 months) in patients with advanced disease. ${ }^{12}$ There were no confirmed objective responses in any of the evaluable patients with cHCC-CCA treated with sorafenib, and most experienced disease progression. This observation aligns with previous reports that $\mathrm{cHCC}$ CCA should be treated similarly to ICC rather than HCC. ${ }^{18,22}$ Comparison of gene expression profiling in HCC, ICC, and cHCC-CCA tumors found that HCC and ICC groups could be clearly distinguished, and that $>70 \%$ of cHCC-CCA tumors clustered with the ICC group, suggesting a closer association of cHCC-CCA to ICC at the gene expression level. ${ }^{22}$

Some studies have demonstrated limited efficacy of both erlotinib and bevacizumab in ICCs. ${ }^{23-25}$ Our cohort included one exceptional responder who received erlotinib and bevacizumab and achieved a complete durable radiographic response. She harbored the EGFR R521K polymorphism; whether this contributed to her remarkable response, however, is unknown. ${ }^{16}$ More experience with these agents in the treatment of cHCC-CCAs is needed before any conclusions can be drawn regarding their efficacy.

In September 2017, the FDA approved the use of nivolumab for HCC in the second-line setting based on the results of the CheckMate 040 open-label tri$\mathrm{al},{ }^{26}$ which demonstrated acceptable tolerability and an objective response rate of $15 \%$ to $20 \%$. More- 
Systemic Therapy for Biphenotypic Cholangiocarcinoma

over, earlier in 2017, the FDA also approved pembrolizumab for MSI-H or MMR-deficient (dMMR) solid tumors that had progressed following prior treatment, based on pooled data from 5 KEYNOTE studies showing a DCR close to $80 \%$. These immunotherapy options were not available when those patients were treated, but could definitely be applied to patients with a significant HCC component or MSI$\mathrm{H} / \mathrm{dMMR}$ status today. It is imperative that better, safer treatments are developed for cHCC-CCAs. Use of next-generation sequencing can help identify additional targetable pathways. Some of our tumor specimens were profiled according to an institutional cancer gene set of the Washington University genomics laboratory, but those results are outside the scope of this review.

Our study has notable limitations. Primarily, it is a retrospective and single-institution study, which can introduce bias. Despite the significant number of noncirrhotic patients for such a rare tumor, there was remarkable provider variation in treatment allocation, dosing, and sequencing. Due to lack of consensus terminology during the study period, no subclassifications were given to the tumors. Furthermore, the effect of tumor heterogeneity on outcomes could not be assessed.

\section{Conclusions}

Patients with cHCC-CCA have a poor prognosis, with a median survival of $<1$ year. Our experience has shown a trend toward better outcomes with gemcitabine-based therapy, especially in combination with a platinum agent, and that this is a reasonable first-line systemic therapy for patients with unresectable or recurrent tumors. Sorafenib monotherapy appears to be ineffective against this malignancy. Genomic analysis of these rare tumors and use of targeted therapies could improve outcomes in the future.

\section{References}

1. Brunt E, Paradis V, Sempoux C, Theise ND. Biphenotypic (hepatobiliary) primary liver carcinomas: the work in progress. Hepat Oncol 2015;2:255273.

2. Jarnagin WR, Weber S, Tickoo SK, et al. Combined hepatocellular and cholangiocarcinoma: demographic, clinical, and prognostic factors. Cancer 2002;94:2040-2046.

3. Wells H. Primary carcinoma of the liver. Am J Sci 1903;126:403-417.

4. Allen RA, Lisa JR. Combined liver cell and bile duct carcinoma. Am J Pathol 1949;25:647-655.

5. Theise ND, Nakashima O, Park YN, Nakanuma Y. Combined hepatocellular-cholangiocarcinoma. In: Bosman FT, Carneiro F, Hruban RH, Theise ND, eds. WHO Classification of Tumours of the Digestive System, 4th ed. Lyon, France: International Agency of Research on Cancer; 2010:225-227.

6. O'Connor K, Walsh JC, Schaeffer DF. Combined hepatocellular-cholangiocarcinoma (cHCC-CC): a distinct entity. Ann Hepatol 2014;13:317322.

7. Maeda T, Adachi E, Kajiyama K, et al. Combined hepatocellular and cholangiocarcinoma: proposed criteria according to cytokeratin expression and analysis of clinicopathologic features. Hum Pathol 1995;26:956-964.

8. Koh KC, Lee H, Choi MS, et al. Clinicopathologic features and prognosis of combined hepatocellular cholangiocarcinoma. Am J Surg 2005; 189:120-125.

9. Lee JH, Chung GE, Yu SJ, et al. Long-term prognosis of combined hepatocellular and cholangiocarcinoma after curative resection comparison with hepatocellular carcinoma and cholangiocarcinoma. J Clin Gastroenterol 2011;45:69-75

10. Yin X, Zhang BH, Qiu SJ, et al. Combined hepatocellular carcinoma and cholangiocarcinoma: clinical features, treatment modalities, and prognosis. Ann Surg Oncol 2012;19:2869-2876.

11. Fowler K, Saad NE, Brunt E, et al. Biphenotypic primary liver carcinomas: assessing outcomes of hepatic directed therapy. Ann Surg Oncol 2015;22:4130-4137.

12. Llovet JM, Ricci S, Mazzaferro V, et al. Sorafenib in advanced hepatocellular carcinoma. N Engl J Med 2008;359:378-390.

13. Hezel AF, Zhu AX. Systemic therapy for biliary tract cancers. Oncologist 2008;13:415-423.

14. Valle J, Wasan $\mathrm{H}$, Palmer DH, et al. Cisplatin plus gemcitabine versus gemcitabine for biliary tract cancer. N Engl J Med 2010;362:1273-1281.

15. Tirkes $T$, Hollar MA, Tann M, et al. Response criteria in oncologic imaging: review of traditional and new criteria. Radiographics 2013;33:1323-1341.

16. Zhou A, Amin M, Fowler KJ, et al. Complete response to erlotinib and bevacizumab in a patient with biphenotypic (hepatobiliary) primary liver carcinoma. J Natl Compr Canc Netw 2015;13:1468-1473.

17. Li R, Yang D, Tang CL, et al. Combined hepatocellular carcinoma and cholangiocarcinoma (biphenotypic) tumors: clinical characteristics, imaging features of contrast-enhanced ultrasound and computed tomography. BMC Cancer 2016;16:158.

18. Lee CH, Hsieh SY, Chang CJ, Lin YJ. Comparison of clinical characteristics of combined hepatocellular-cholangiocarcinoma and other primary liver cancers. J Gastroenterol Hepatol 2013;28:122-127.

19. Li JS, Han TJ, Jing N, et al. Obesity and the risk of cholangiocarcinoma: a meta-analysis. Tumour Biol 2014;35:6831-6838.

20. Noureddin M, Rinella ME. Nonalcoholic fatty liver disease, diabetes, obesity, and hepatocellular carcinoma. Clin Liver Dis 2015;19:361-379.

21. Gebbia V, Giuliani F, Maiello E, et al. Treatment of inoperable and/or metastatic biliary tree carcinomas with single-agent gemcitabine or in combination with levofolinic acid and infusional fluorouracil: results of a multicenter phase II study. J Clin Oncol 2001;19:4089-4091.

22. Woo HG, Lee JH, Yoon JH, et al. Identification of a cholangiocarcinoma-like gene expression trait in hepatocellular carcinoma. Cancer Res 2010;70:3034-3041.

23. Jang JS, Lim HY, Hwang IG, et al. Gemcitabine and oxaliplatin in patients with unresectable biliary cancer including gall bladder cancer: a Korean Cancer Study Group phase II trial. Cancer Chemother Pharmacol 2010;65:641-647.

24. Lubner SJ, Mahoney MR, Kolesar JL, et al. Report of a multicenter phase II trial testing a combination of biweekly bevacizumab and daily erlotinib in patients with unresectable biliary cancer: a Phase II Consortium study. J Clin Oncol 2010;28:3491-3497.

25. Zhu AX, Meyerhardt JA, Blaszkowsky LS, et al. Efficacy and safety of gemcitabine, oxaliplatin, and bevacizumab in advanced biliary-tract cancers and correlation of changes in 18-fluorodeoxyglucose PET with clinical outcome: a phase 2 study. Lancet Oncol 2010;11:48-54.

26. El-Khoueiry AB, Sangro B, Yau T, et al. Nivolumab in patients with advanced hepatocellular carcinoma (CheckMate 040): an open-label, non-comparative, phase $1 / 2$ dose escalation and expansion trial. Lancet 2017;389:2492-2502. 